\title{
Exaggerated Distal Medial Arm Depression after Brachioplasty: A Newly Described Post-Brachioplasty Deformity and a Modification of Major Brachioplasty Operation to Prevent it
}

\author{
SHERINE M. ABOUL FOTOUH, M.D. and NAHED SAMIR, M.D. \\ The Department of Plastic, Burn and Maxillofacial Surgery, Faculty of Medicine, Ain Shams University, Cairo, Egypt
}

\begin{abstract}
Background: The dramatic progress in the surgical treatment of obesity increases the demand for post-massive weight loss (MWL) corrective operations including brachioplasty. We describe a post-brachioplasty deformity not yet been described in the literature. There is a naturally located anteromedial depression of the distal third of the arm. This depression is exacerbated after brachioplasty resulting in contour deformity. A suggestive modification of major brachioplasty operation to avoid this deformity is also studied.
\end{abstract}

Patients and Methods: This is a prospective study of 15 MWL patients, 39-54 years old with advanced upper arm sagging reaching the elbow. As a modification of major brachioplasty, a triangular dermofat flap is created and buried under the depression of the distal third of the arm aiming to restore the roundness and firmness of this part. Pre- and postoperative photographs are taken. Follow-up period ranged from 6-12 months.

Results: No contour deformity was recorded. Complication rate was $10 \%$ in the form of delayed healing of one axilla and hypertrophic scars of both sides in another patient.

Conclusion: This modification is useful especially for thin and elderly patients who are most likely exposed to this unacceptable deformity.

Key Words: Brachioplasty - Modification.

\section{INTRODUCTION}

The extraordinary rise of brachioplasty continues to be an important part of addressing the total solution for MWL patients [1].

Since brachioplasty was first described in the 1920s [2], techniques have evolved to include liposuction, excisional surgery, a combination of liposuction and excision, single-versus multi-staged procedures and, depending on the characteristics of the deformity, even implants [3-12]. Lockwood emphasized the importance of secure tightening of the superficial fascial system resulting in a smoother contour, tighter closure and finer scar [14].

In severe arm redundancy, post-operative imperfect contour isn't uncommon. Under-reduction, over-reduction, constriction arm band and depression deformity are different forms of unaccepted contour defects. According to Wendy Chen et al., the constriction arem band deformity is a preoperative finding that can't be corrected by brachioplasty and even exacerbated post-operatively in $50 \%$ of cases. It is associated with higher current BMI and larger resection weights. Most bands were located in the middle $(26 \%)$ or distal-third $(74 \%)$ of the upper arm and found bilaterally $(68 \%)$ [15].

In our study, another post-brachioplasty contour deformity, not yet mentioned in the literature, is described. It's a triangular depression deformity of the lower medial arm few centimeters proximal to the medial epicondyle (Fig. 1). It's an exaggeration of a naturally present mild depression due to lack of soft tissue support at that area (Fig. 2). The lower central part of the arm is occupied by the biceps muscle anteriorly and brachialis muscle posteriorly while the lower lateral part has the origin of brachioradialis muscle leaving the medial lower arm without significant soft tissue support. After brachioplasty of severe arm redundancy, this depression is highlighted and look like local strangulation of the arm. This unaccepted deformity gives the impression of thinner arm in relation to the forearm because it affects the most distal part of the arm.

We also suggested a prophylactic modification of major brachioplasty operation [18], especially in severe arm redundancy, elderly and thin patients. We selected MWL patients with severe arm redundancy who were expected to be more prone to the deformity after brachioplasty. They were good candidates for major brachioplasty because of severe arm redundancy extending from the elbow to the axilla. They had no lateral chest skin redundancy or not interested in its correction. 

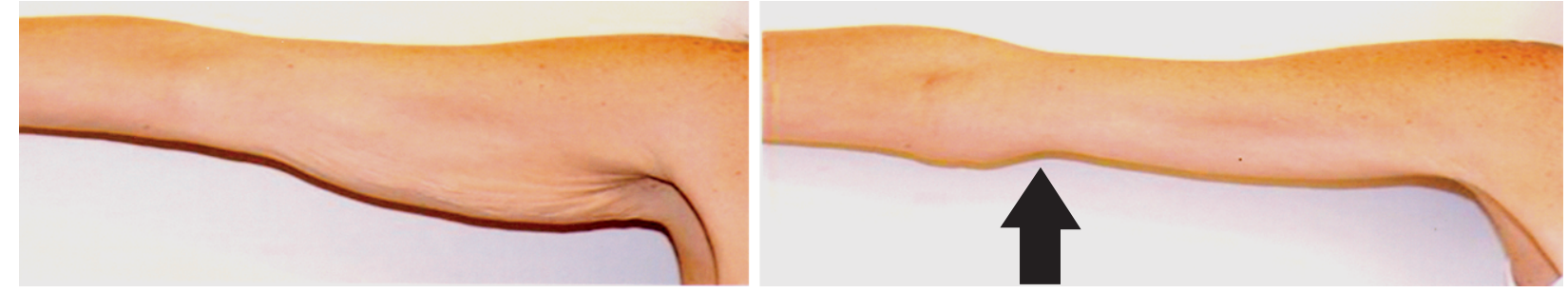

Fig. (1): (A): Arm redundancy. (B): Post-brachioplasty depression deformity (black arrow) [16].

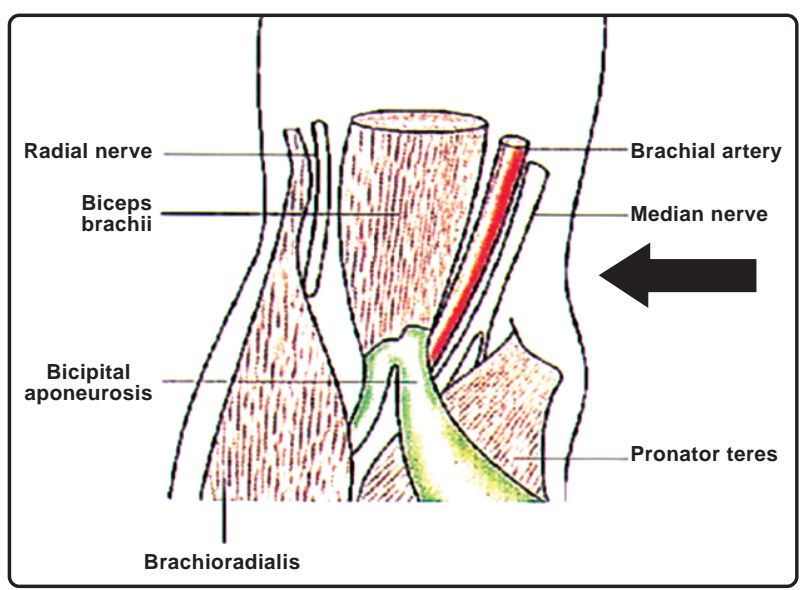

Fig. (2): Cubital fossa anatomy showing the natural lower medial depression of the arm (black arrow) due to lack of soft tissue support at that area superior to the medial epicondyle bonny prominence [17].

\section{PATIENTS AND METHODS}

Fifteen MWL patients with severe arm redundancy underwent major brachioplasty in the period from 2016-2018 were involved in this study. They were all females, 39-54 years old. Pre-operative assessment included weight loss, BMI, general condition, history of chronic diseases, routine laboratory investigations, skin tone evaluation, degree of redundancy and pinch test to quantify the subcutaneous fat of the arm circumferentially. Any pre-operative contour deformity was documented. Informed consent was signed.

Markings were drawn the day of the surgery while the patient standing, with the arms abducted to 90 degrees and elbow fully extended. The superior resection line was drawn connecting the points representing axillary apex, bicipital groove and medial epicondyle. The skin amount to be removed was estimated through pinching the sagging medial skin between the thumb and index fingers along the line drawn. Now, the inferior resection line could be marked meeting the superior line at the medial epicondyle point and extending proximally to the axilla. The proximal ends of the superior and inferior resection lines at the axilla would be further extended in the axillary hollow and met at a point according to the skin laxity. Transverse hatched lines were drawn across the resection ellipse to guide skin closure. Areas for liposuction were marked too. The plan was to avoid over resection and tight closure in the distal arm especially, the distal third while perform quite tight closure of the proximal arm. The markings should represent this plan.

Markings of the expected deformity area: A triangular depression can be identified on the antero-medial aspect of the arm few centimeters proximal to the cupital area (T) (Fig. 3). This triangle together with a mirror image triangle ( $\left.\mathrm{T}^{\prime}\right)$ on the resection skin ellipse was marked.

\section{Technique:}

The patient was placed in supine position with arms abducted 90 degrees on arm table with feasibility for full abduction during surgery. Intravenous line and blood pressure cuff were placed in the lower limb. General anaesthesia was a necessity for major brachioplasty. Both upper limbs were prepared and draped routinely with hands and distal forearms covered with sterile stockinette.

Infiltration of superwet fluid in posterior and medial arm areas was done. Amount of liposuction of posterior arm varied according to the degree of lipodystrophy of each patient. Liposuction of medial arm aimed to loosening the dissection plane and providing tension free closure.

Before excision of redundant tissue, the triangle T' was de-epithelialized and dissected as a dermofat flap with its base at the superior line (Fig. 4). Undermining of triangle $\mathrm{T}$ at the subcutaneous plane transformed the 2 triangles into a continuous diamond shape flap with its lower triangular half de-epithelialized. T' was folded superiorly deep to $\mathrm{T}$ and fixed to its through a pull out suture. This technique aimed to augment the triangular depression (T) and prevent any possible depression deformity. 
The excision started by incising the superior line of the ellipse (except across the diamond flap) down to the superficial fascial system (SFS). Dissection was carried downwards deep to SFS using the cutting cautery. Continuous re-evaluation by pulling the free edge of the dissected flap upwards and marking for resection was done to achieve the proper tightness and contouring. The excision was performed from distal to proximal with stapling of the skin edges to close the wound and control the edema after meticulous hemostasis. The SFS was sutured with $2 / 0$ vicryl followed by deep dermal sutures with $3 / 0$ vicryl then, sub-cuticular closure with 4/0 monocryl. No drains were needed (Fig. 5).

Pre- and post-operative standard medical photographs were taken. Patients were asked to evaluate their final results regarding arm size, contour and symmetry together with quality and position of the scar using a four-point numerical rating scale, where 0 indicated no improvement and 3 indicated highly satisfied. In addition, patients were objectively evaluated by 2 plastic surgeons who were not involved in the treatment. These surgeons compared pre- and post-operative (one month and 6 months) photographs with regard to size, contour, symmetry and quality and position of the scar. Their evaluation was recorded as percentage of improvement on a quartile grading scale: <25\%: Mild improvement; 25-50: Moderate improvement; 51-75\%: Good improvement and 76100\%: Excellent improvement.

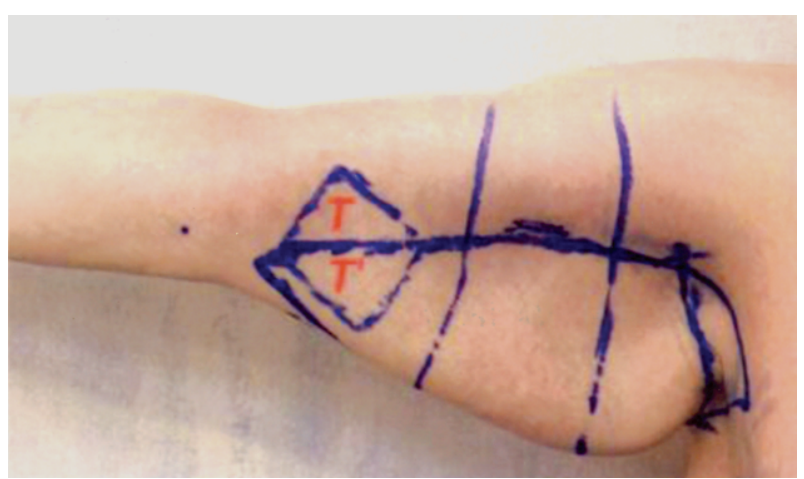

Fig. (3): Pre-operative marking: The excision ellipse, T representing the natural depression in lower medial arm, T' representing the mirror image triangle.
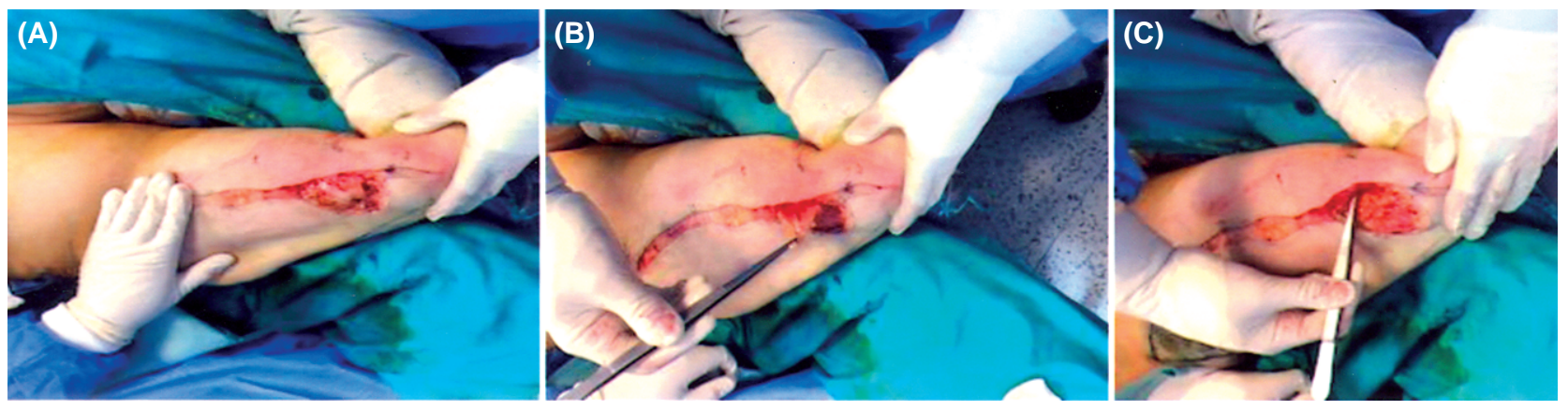

Fig. (4): (A): Triangle T' deepithelialized. (B): Dissected as dermofat flap. (C): Buried under undermined T.

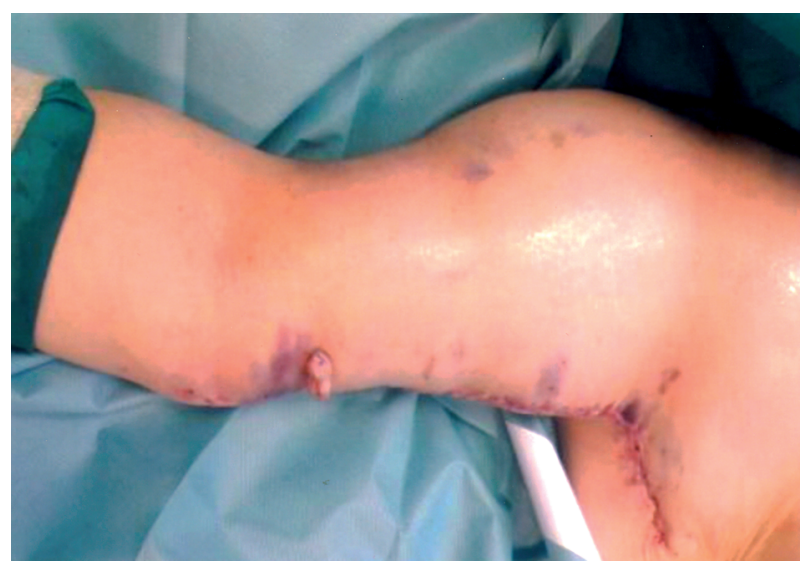

Fig. (5): Pull out suture and closure.

\section{RESULTS}

Fifteen female patients with severe arm redundancy after MWL were operated upon using the modified major brachioplasty in the period from
2016-2018. Their average age was 49.6 years (range $39-54$ years). The average weight loss was 33 kilograms (range 27-51 kilograms) with stable body weight for at least 6 months. The average follow-up period was 7.6 months.

Arm size, contour and symmetry together with quality and position of the scar were the parameters for results assessment. Early and late complications were also documented.

No complaint regarding arm size, contour or symmetry or scar position was recorded with subjective evaluation revealing an overall high degree of satisfaction in 13 of 15 patients (86.7\%). Two patients $(13.3 \%)$ reported moderate satisfaction (Figs. 6,7).

The objective aesthetic outcome was rated as excellent in 12 patients $(80 \%)$ and good in the remaining 3 patients (20\%). 


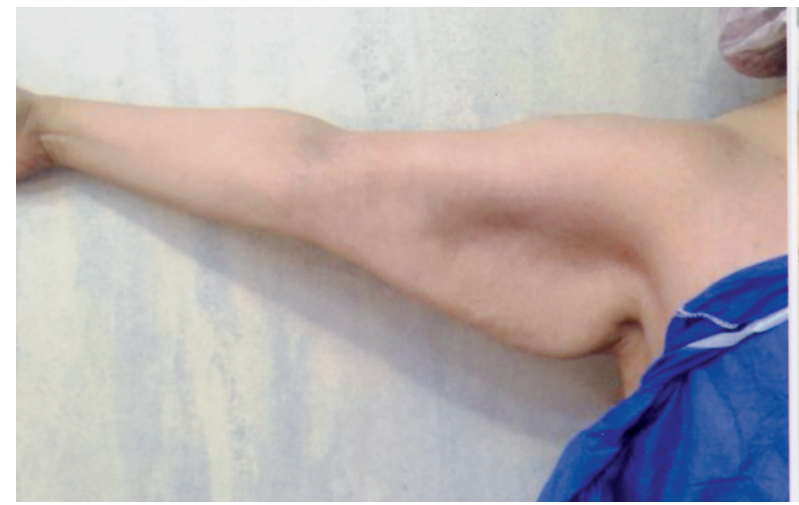

Fig. (6A): Pre-operative right arm (front).

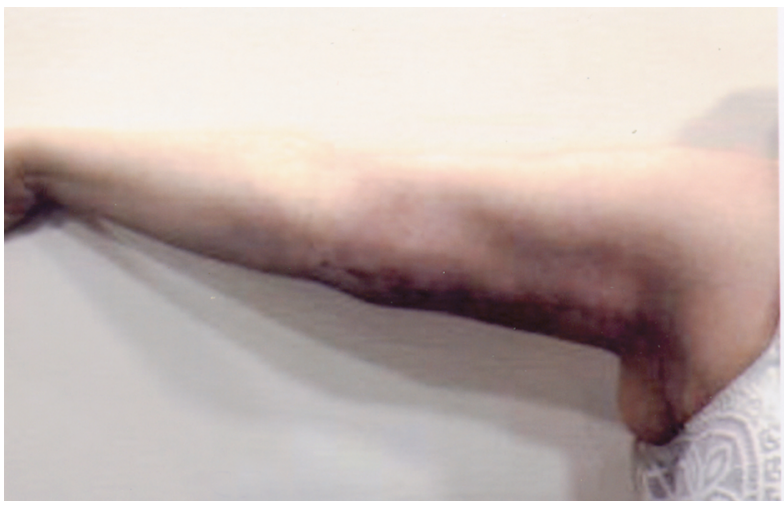

Fig. (6C): Post-operative right arm (front).

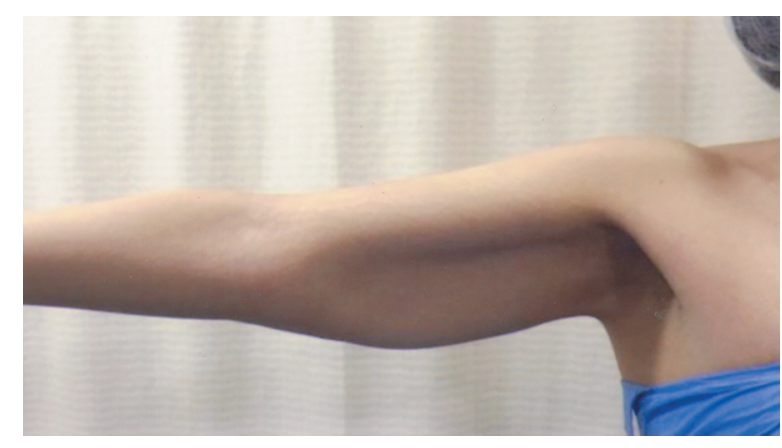

Fig. (7A): Pre-operative right arm (front).

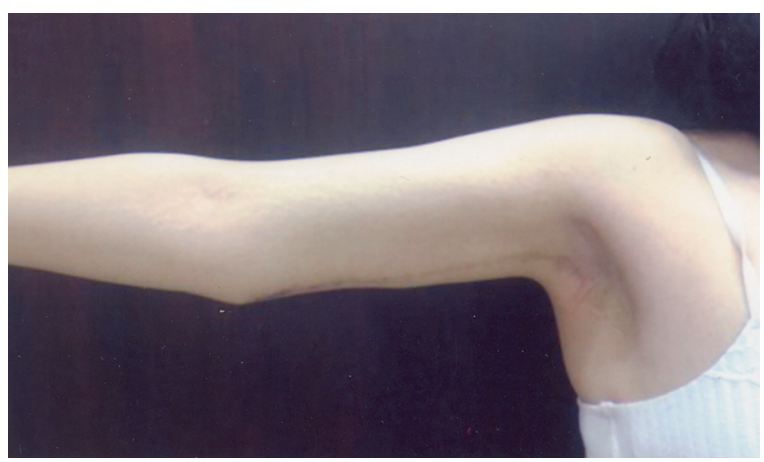

Fig. (7C): Post-operative right arm (front).

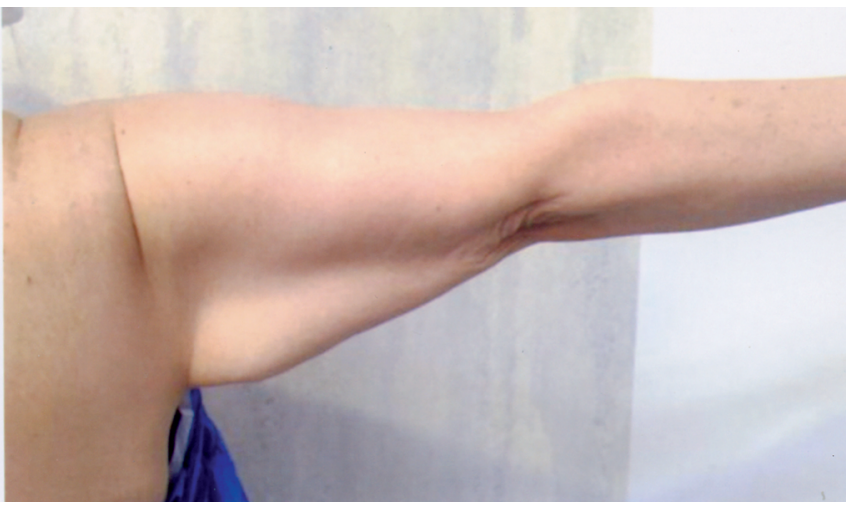

Fig. (6B): Pre-operative right arm (back) of 54 years old patient.

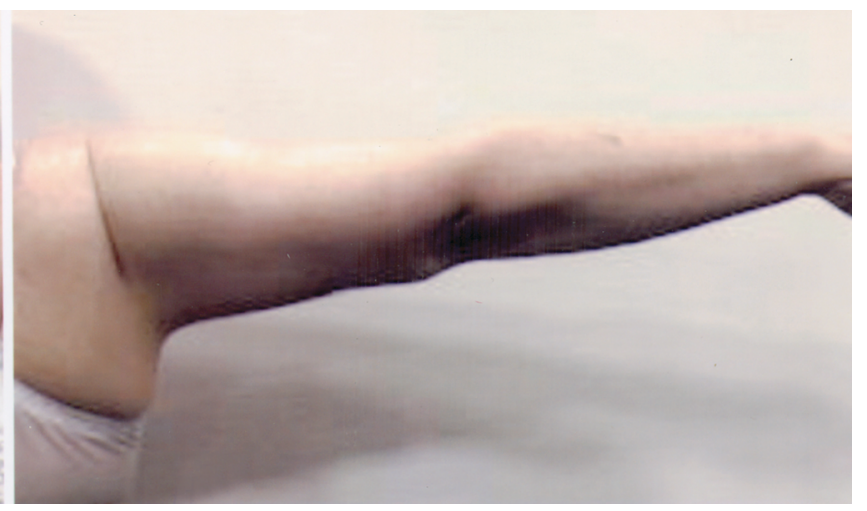

Fig. (6D): Post-operative right arm (back).

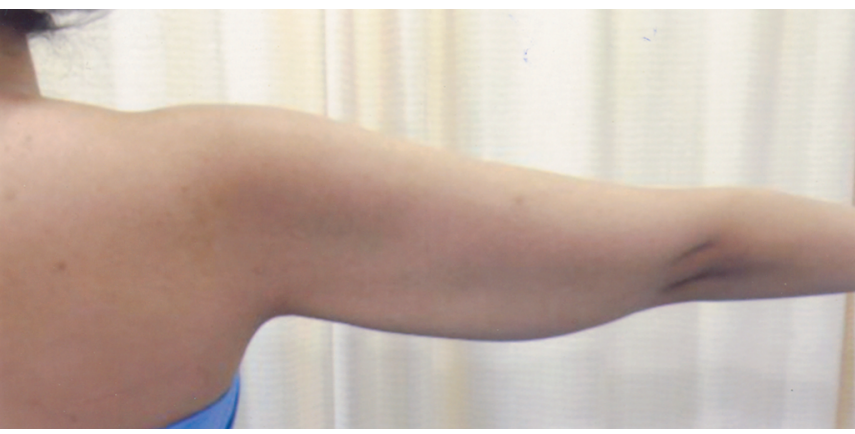

Fig. (7B): Pre-operative right arm (back) of 40 years old patient.

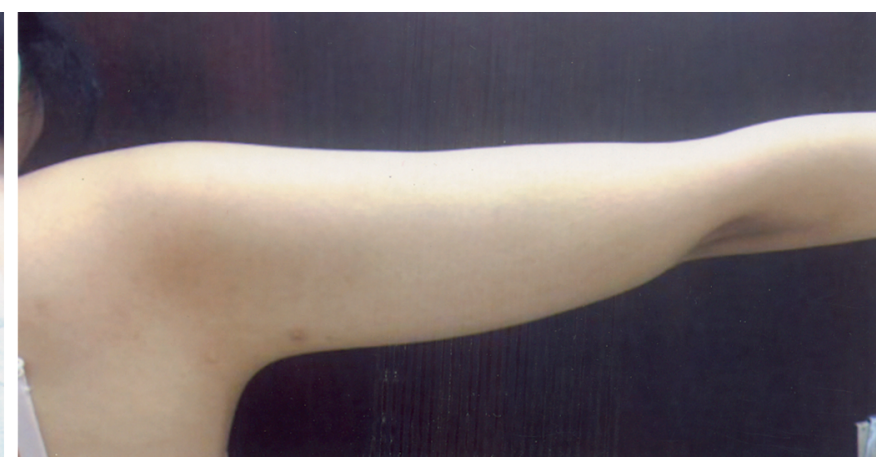

Fig. (7D): Post-operative right arm (back). 
One patient showed delayed healing in one axilla (3.3\%). The late complication was bilateral hypertrophic scar in another patient (6.7\%) (Table 1).

No re-operation was needed to correct any of the complications.

Table (1): Complications.

\begin{tabular}{ll}
\hline Complication & Number \\
\hline Delayed healing & $1(3.3 \%)$ \\
Hypertrophic scars & $2(6.7 \%)$ \\
\hline Total & $3(10 \%)$ \\
\hline
\end{tabular}

\section{DISCUSSION}

Brachioplasty procedures have steadily improved and now are routinely reaching patients' goals. In markedly sagging arms, it's somewhat more difficult to produce a truly tight and perfectly contoured result as well as an absolutely fine scar. This is probably because these patients usually have poor skin tone and elasticity [18].

In 2015, we had two patients with postbrachioplasty contour deformity. The deformity was a triangular depression in the lower medial third of the arm, few centimeters proximal to the medial epicondyle. This was expressed by the patients as "My arms became thinner than my forearms". This complaint was other patients' fear who had their friends or relatives underwent brachioplasty by other plastic surgeons and have the same deformity. Although, lipofilling of the depressed area treated the deformity efficiently, it wasn't an uncommon problem and had to have a solution.

This described deformity is different from the arm band deformity that has been mentioned at many national meetings and forums and lastly reported in the literature by Wendy Chen et al., [15].

Actually, this triangular depression is a naturally present depression due to lack of soft tissue support proximal to a bony prominence (medial epicondyle) which is accentuated after brachioplasty. To avoid this deformity, conservative liposuction and skin excision at that site was the prophylactic plan. This succeeded in preventing the depression deformity but resulted in loss of the taught, round and esthetic contour of the lower third of the arm.

Goddio, described a brachioplasty technique in which he de-epithelialize the skin excess ellipse along the entire arm instead of excising it [7]. $\mathrm{He}$ buried the de-epithelialized tissue; after dissecting it as a posteriorly based flap; in an undermined anterior pocket to provide esthetic arm fullness and proper tightness which might be helpful in the very thin arm and elderly patients. His clinical series was 12 elderly thin women, only 3 of them had lost weight; the remainder had never been obese.

It's not difficult to restore the proper contour in the proximal arm with the traditional excision technique due to the presence of adequate soft tissue support all through the way. We confined the use of the dermofat flap to the distal third of the arm in our modification of major brachioplasty operation. The dermofat flap is anteriorly based providing the flexibility to readjust the extent of excision posteriorly. Inserting the flap in its pocket by folding it, further augments the incision line.

Brachioplasty as one of the body contouring operations in MWL patients can be associated with post-operative complications such as hematoma, seroma, infection, delayed healing, unfavorable scars and contour deformities. In the literature, the rate of complications varied widely from $1.3 \%$ up to $56 \%$ [19-21]. Other rare serious complications include fluid overload, thromboembolism, fat embolism and cardiopulmonary dysfunction most frequently occurring if brachioplasty is performed in conjunction with other body contouring procedures [7,8,22-24].

In our study, no complaint regarding arm size, contour or symmetry or scar position was recorded. The subjective and objective evaluations generally showed good to excellent results. The rate of complications was $10 \%$. One patient showed delayed healing in one axilla (3.3\%) and another patient had bilateral hypertrophic scar as a late complication $(6.7 \%)$. No re-operation was needed to correct any of the complications.

On combining body contouring surgeries, we have to consider the time factor, presence of any co-morbidity, patient's age and amount of liposuction to fulfill the safety measures and avoid any serious medical complications.

\section{Conclusion:}

In our current study, we described a postbrachioplasty depression deformity not yet described in the literature. We also presented a modification of the major brachioplasty operation aiming to prevent this potential depression deformity in severe arm redundancy in post MWL patients. The modified technique was successfully applied with satisfying arms contour and reasonable complication rate. 


\section{REFERENCES}

1- SepehrEgrari: Brachioplasty: A personal approach. Aesthetic Plast. Surg., 36 (2): 193, 2016.

2- Toole J.P., Song A. and Rubin J.P.: The history of body contouring surgery. Semin in Plast. Surgery, 201: 5, 2006.

3- Abboud M.H., Abboud N.M. and Dibo S.A.: Brachioplasty by power-assisted liposuction and fat transfer: A noval approach that obviates skin excision. Aesthet. Surg. J., 36 (8): 908, 2016.

4- Teimourian B. and Malekzadeh S.: Rejuvenation of the upper arm. Plast. Reconstr. Surg., 102: 545, 1998.

5- Richards M.E.: Minimal incision brachioplasty: A first choice option in arm reduction surgery. Aesthet. Surg. J., 21: 301, 2001.

6- Lockwood T.: Contouring of the arms, trunk and thighs. Plastic surgery: Indications, operations and outcomes Vol. 5: Aesthetic surgery. Achauer B.M., Eriksson E., Gjyuron B., et al., eds. St. Louis, MO: Mosby, 2000.

7- Goddio A.S.: A new technique for brachioplasty. Plast. Reconstr. Surg., 84: 85, 1989

8- Gililand M.D. and Lyos A.T.: CAST Liposuction of the arm improves aesthetic results. Aesthet. Plast. Surg., 21: $225,1997$.

9- Gililand M.D. and Lyos A.T.: CAST liposuction: An alternative to brachioplasty. Aesthet. Plast. Surg., 21: 398, 1997.

10- Vogt P.A.: Brachial suction assisted lipoplasty and brachioplasty. Aesthet. Surg. J., 21: 163, 2001.

11- Nguyen A.T. and Rohrich R.J.: Liposuction assisted posterior brachioplasty: Technical refinements in upper arm contouring. Plast. Reconstr. Surg., 126: 1365, 2010.

12- Abadesso I. and Serra F.: Augmentation brachioplasty: Surgery for improving the appearance of the arms. Rev. Bras. Cir. Plast., 27: 97, 2012.

13- Ferraro G.A., De Francesco F., Razzano S., et al.: Modified fish-incision technique in brachioplasty: A surgical approach to correct excess skin and fat of the upper arm (restoring the armpit contour). Aesthet. Plast. Surg., 39 (2): 203, 2005.

14- Lockwood T.: Brachioplasty with superficial fascial system suspension. Plast. Reconstr. Surg., 96: 912, 1995.

15- Wendy Chen, Isaac B. James, Jeffrey A. Gusenoff, J. Peter Rubin: The constriction arm band deformity in brachioplasty patients: Characterization and incidence using a prospective registry. Plast. Reconstr. Surg. Accepted Advance Online Article, 2018.

16- http://www.drmoses.com/wp-content/uploads/brachioplasty -01a-rightjpg

17- http://image1.slideserve.com/246861/contents-of-theright-cubital-fossa-n.jpg.

18- Richard A. Mladick: Arm lift. The art of aesthetic surgery, principles and techniques. Vol. 6: Aesthetic surgery. Foad Nahai, eds. Paces Plastic Surgery, Atlanta, Georgia, 2005.

19- Zomerli T.A., Neaman K.C., Armstrong S.D., et al.: Brachioplasty outcomes: A Review of a multipractice cohort. Plast. Reconstr. Surg., 131: 883, 2013.

20- Bossert R.P., Dreifuss S., Coon D., et al.: Liposuction of the arm concurrent with brachioplasty in the massive weight loss patient: Is it safe? Plast. Reconstr. Surg., 131: $357,2013$.

21- De Runz A., Colson T., Minetti C., et al.: Liposuction assisted medial brachioplasty after massive weight loss: An efficient procedure with a high functional benefit. Plast. Reconstr. Surg., 135: 74e, 2015.

22- Knoetgen J.3rd and Moran S.L.: Long-term outcomes and complications associated with brachioplasty: A retrospective review and cadaveric study. Plast. Reconstr. Surg., 117: 2219,2006

23- Gusenoff J.A., Coon D. and Rubin J.P.: Brachioplasty and concomitant procedures after massive weight loss: A statistical analysis from a prosective registry. Plast. Reconstr. Surg., 122: 595, 2008.

24- Garcia Botero A., Garcia Wenninger M. and Fernandez Loaiza D.: Complications after body contouring in postbariatric patients. Ann. Plast. Surg., 79 (3): 293, 2017. 